\title{
BMJ Open Anaemia and related nutrient deficiencies after Roux-en-Y gastric bypass surgery: a systematic review and meta-analysis
}

Ting-Chia Weng, ${ }^{1}$ Chia-Hsuin Chang,,${ }^{1,2,3}$ Yaa-Hui Dong, ${ }^{4}$ Yi-Cheng Chang, ${ }^{2,5}$ Lee-Ming Chuang ${ }^{1,2,3}$

To cite: Weng T-C, Chang $\mathrm{C}-\mathrm{H}$, Dong $\mathrm{Y}-\mathrm{H}$, et al. Anaemia and related nutrient deficiencies after Roux-en-Y gastric bypass surgery: a systematic review and meta-analysis. BMJ Open 2015;5:e006964. doi:10.1136/bmjopen-2014006964

- Prepublication history and additional material is available. To view please visit the journal (http://dx.doi.org/ 10.1136/bmjopen-2014006964).

T-CW and C-HC contributed equally.

Received 20 October 2014 Revised 30 May 2015 Accepted 15 June 2015

CrossMark

For numbered affiliations see end of article.

Correspondence to Dr Lee-Ming Chuang; leeming@ntu.edu.tw

\section{ABSTRACT}

Objective: To obtain a pooled risk estimate on the long-term impact of anaemia and related nutritional deficiencies in patients receiving Roux-en-Y gastric bypass (RYGB) surgery.

Design: Systematic review and meta-analysis.

Data sources: MEDLINE, EMBASE and Cochrane databases were searched to identify English reports published before 16 May 2014.

Eligibility criteria: Articles with case numbers $>100$, follow-up period $>12$ months, and complete data from both before and after surgery were selected. Outcomes of interest were changes in baseline measurements of proportion of patients with anaemia, by haemoglobin, haematocrit, ferritin, iron, vitamin $B_{12}$ and folate levels.

Data collection and analysis: Two reviewers independently reviewed data and selected six prospective and nine retrospective studies with a total of 5909 patients. A random effect model with inverse variance weighting was used to calculate summary estimates of outcomes at 6, 12, 24 and 36 months postoperatively.

Results: Proportion of patients with anaemia was $12.2 \%$ at baseline, which, respectively, increased to $20.9 \%$ and $25.9 \%$ at 12 and 24 months follow-up, consistent with decreases in haemoglobin and haematocrit levels. Although the serum iron level did not change substantially after surgery, the frequency of patients with ferritin deficiency increased from $7.9 \%$ at baseline to $13.4 \%$ and $23.0 \%$ at 12 and 24 months, respectively, postoperation. Vitamin $B_{12}$ deficiency increased from $2.3 \%$ at baseline to $6.5 \%$ at 12 months after surgery in those subjects receiving RYGB. There was no obvious increase in folate deficiency.

Conclusions: RYGB surgery is associated with an increased risk of anaemia and deficiencies of iron and vitamin $B_{12}$, but not folate. Ferritin is more sensitive when serum iron level is within normal range.

\section{INTRODUCTION}

Obesity is a complex metabolic disorder that is often associated with related comorbid

\section{Strengths and limitations of this study}

- This meta-analysis summarised the late outcomes of haematological changes and relevant nutritional deficiencies after Roux-en-Y gastric bypass (RYGB) by including a large sample and exclusion of small trials.

- In our meta-analysis, there is not much uncertainty as to the effect of RYGB on development of anaemia, low ferritin and vitamin $B_{12}$ deficiencies.

- Our findings can be translated into clinical practice by implementing preoperation and postoperation surveillance of ferritin and vitamin $B_{12}$ levels, and nutrient supplementation.

- This study is limited by heterogeneity across studies without formal quality assessment.

conditions that require medical intervention, including cardiovascular disease, type 2 diabetes mellitus, dyslipidaemia, degenerative osteoarthritis and psychological disabilities, and pose a huge social burden. ${ }^{1}$ The increasing prevalence and severity of obesity likely results from complex interactions of dietary intake, physical activity, genes and the environment. $^{2}{ }^{3}$ However, conventional medical treatment and lifestyle modification seldom yield satisfactory long-term results in obese people. $^{4-6}$ After more than 50 years' experience performing bariatric surgery, the procedure is now recommended for morbidly obese individuals, with proven long-lasting effects on weight reduction, remission of diabetes and cardiovascular complications and mortality. ${ }^{7-11}$

Over the past decades, Roux-en-Y gastric bypass (RYGB) has been a classical bariatric surgery with $85 \%$ of patients receiving this restrictive/malabsorptive procedure. ${ }^{12} 13 \mathrm{In}$ this procedure, gastric capacity is reduced by $90-95 \%$. However, adverse effects such as 
nutritional deficiencies and gastrointestinal symptoms have been observed following RYGB, including disturbances of anatomical and physiological functions. ${ }^{14}$ A wide range of prevalence and severity of anaemia after RYGB surgery has been reported and the related causes are not yet fully understood. ${ }^{15-18}$ Therefore, we conducted a systematic review and meta-analysis of all published studies to obtain a pooled risk estimate of anaemia and to summarise the reported nutritional deficiencies relevant to anaemia during long-term follow-up of patients receiving RYGB surgery.

\section{METHODS}

The methods for this systematic review and meta-analysis were developed according to recommendations from the Preferred Reporting Items for Systematic Reviews and Meta-Analyses (PRISMA), ${ }^{19}$ and the Meta-Analysis of Observational Studies in Epidemiology statements. ${ }^{20}$

\section{Identification of trials}

We applied Medical Subjects Heading (MeSH) terms for keyword and text word searching with 'gastric bypass' as intervention term, and subject terms including 'anemia', 'hemoglobins', 'hematocrit', 'anemia, iron-deficiency', 'iron', 'ferritins', 'vitamin $\mathrm{B}_{12}$ ', 'vitamin $\mathrm{B}_{12}$ deficiency', 'folic acid', 'folic acid deficiency' and 'folate'. We searched all fields in MEDLINE (via PubMed), all text in Cochrane Library (including the Cochrane Database of Systematic Reviews; the Database of Abstracts of Reviews of Effects; and the Cochrane Register of Controlled Trials) and searched 'Roux-en-Y gastric bypass' in ClinicalTrials.gov website (https://register. clinicaltrials.gov/). The search was last updated on 16 May 2014 (see search query in online supplementary appendix 1). The references of review articles on surgical treatment of obesity were examined manually to identify additional articles that were not found in the computerised databases. ${ }^{17} 18$ 21-26

\section{Eligibility criteria}

This meta-analysis targeted studies on treatment of morbid obesity patients (class III with body mass index $(B M I) \geq 40$; or $>35$ with comorbidities) using standard RYGB procedure. We included both laparoscopy and traditional open procedure. We included randomised controlled trials, case series, as well as observational studies that enrolled diabetic and non-diabetic patients. Studies were required to provide any outcome of (1) anaemia and anaemia-related parameters (haemoglobin, haematocrit) and/or (2) the relevant nutrient profiles including serum iron, ferritin, vitamin $\mathrm{B}_{12}$ or folate. Information of therapeutic and/or prophylactic nutrient supplementations was allowed to be included.

We excluded non-English articles and studies with gastric bypass surgeries carried out by procedures other than RYGB, patients aged $<18$ years and studies with fewer than 100 participants. The threshold number of
100 was selected for practicality and to avoid the small study effects potentially distorting results of metaanalyses. Reports that did not present preoperation baseline data, or follow-up duration less than 12 months, were also excluded. Figure 1 illustrates the literature search and study selection.

\section{Outcomes of interest}

The primary outcomes of interest in this meta-analysis were the proportions of patients who had anaemia, and iron, ferritin, folate and vitamin $\mathrm{B}_{12}$ deficiencies, after the surgery and the changes in percentage of the above outcomes from the baseline after more than 12 months follow-up. The secondary outcomes of interest included the mean haematocrit, haemoglobin, serum iron, ferritin, folate and vitamin $B_{12}$ levels, and the changes in the above measurements from baseline. We did not define specific cut-off values for related nutrient deficiencies because different criteria were used in different studies.

\section{Data extraction}

Two physician reviewers (C-H C, T-C W) independently evaluated each identified study and extracted relevant characteristics, including quality of the studies. Disagreement on specific studies between the two reviewers was resolved through discussion. Extracted information included study characteristics (author, published year, study design and intended follow-up duration); patient characteristics (country, mean or median age, percentage of males and mean BMI); and number of study participants and outcomes. The quality of each study was assessed based on the proportion of participants missing during follow-up. The means of outcome measurements and proportions of deficiencies at baseline, and 6, 12, 24 and 36 months after surgery, were extracted. Outcomes reported at different time points other than the stated time frame were combined with the nearest time point. The changes in these measurements from the baseline $(\Delta \mathrm{Ct})$ were also calculated as (Ct-Cb), with $\mathrm{Ct}$ and $\mathrm{Cb}$ being the outcome measurements at specific time points, and at baseline if data were available.

\section{Statistical analysis}

For bariatric surgeries, there are substantial differences in preoperative treatment, RYGB procedures, and postoperative nutritional management and outcome monitoring. Accordingly, we applied the random effects model with an inverse variance weighting method to capture variations across studies, and calculated the pooled estimates for the outcomes of interest. For each outcome at specific time points, we calculated the summary estimates and their 95\% CIs based on data from the total number of available participants at that time point. A continuity correction factor of 0.01 was used for studies that reported zero incidence for the outcomes. Lower bounds of the CIs smaller than zero were 
Figure 1 Literature search and study selection.

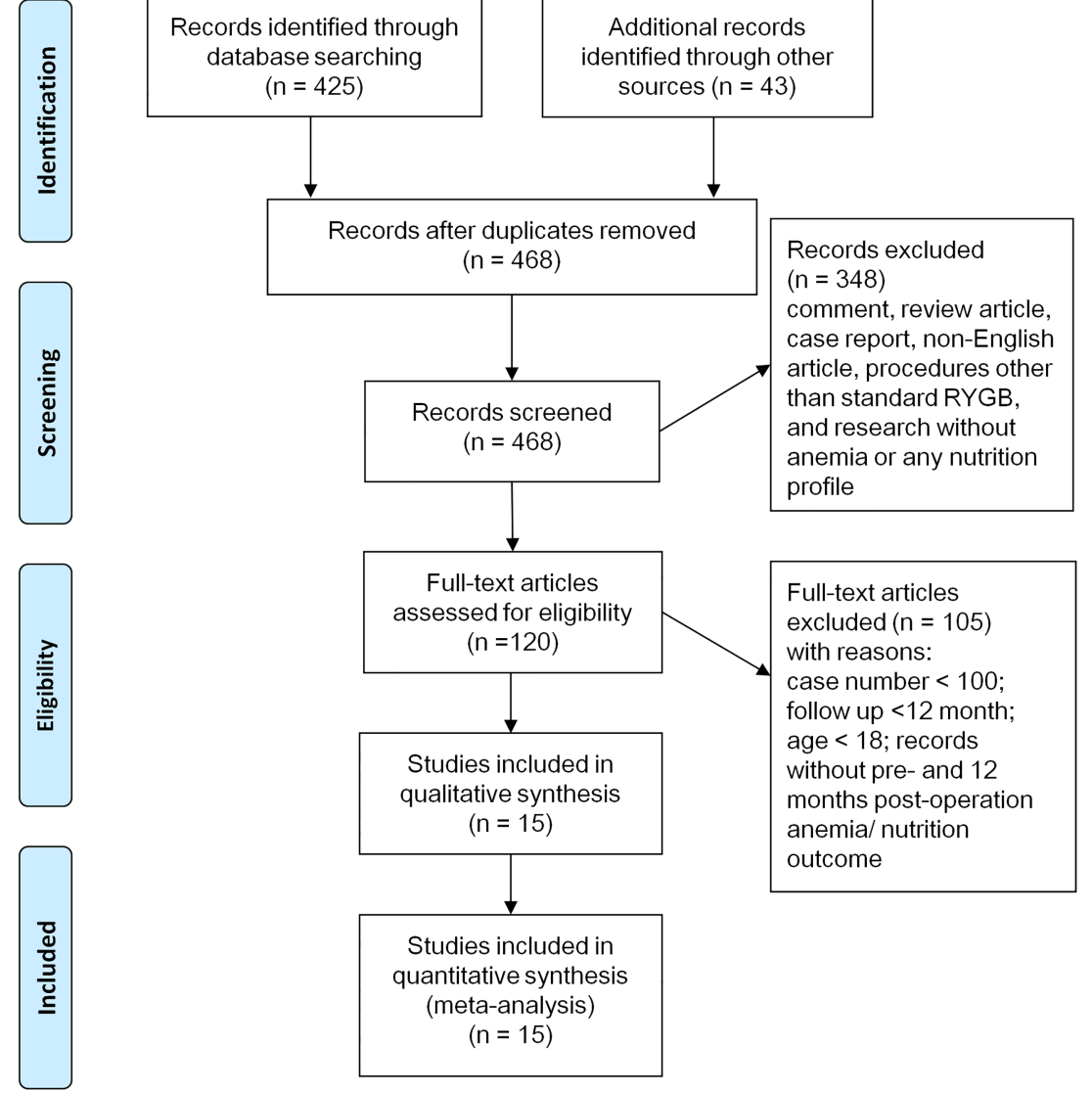

reframed at zero. For changes in outcome measurements at each time point from the baseline, the summary estimates with 95\% CIs were computed according to the absolute difference $(\Delta \mathrm{Ct})$ and corresponding variance $[\operatorname{Var}(\Delta \mathrm{Ct})]$ in each study, where $[\operatorname{Var}(\Delta \mathrm{Ct})]$ was indirectly estimated as $[\operatorname{Var}(\mathrm{Ct})+\operatorname{Var}(\mathrm{Cb})]$, given that none of the studies provided the original data on SD. Statistical heterogeneity was evaluated by the $\mathrm{I}^{2}$ statistic, with a value of $50 \%$ or more illustrating a substantial level of heterogeneity. Publication bias was assessed by the funnel plot, Begg's test and Egger's test. STATA V.9.0 (StataCorp, College Station, Texas, USA) was used for the analyses. A two-sided $p$ value of 0.05 was defined as statistical significance.

\section{RESULTS}

We identified 468 English studies on RYGB surgeries for patients with obesity from computerised literature databases and reference lists of systematic reviews (figure 1). A total of 453 studies were excluded because they were review articles, research without nutrition profiles or relevant outcomes, procedures other than RYGB, or the number of subjects was less than 100 . A total of 15 observational studies enrolling morbidly patients with obesity were included in this meta-analysis. ${ }^{27-41}$ The number of study subjects ranged from 100 to 1125 , totalling 5909 patients (table 1). Most of the studies were conducted in the USA; six were prospective. ${ }^{27} 28 \quad 32 \quad 36 \quad 3741$ Four studies had an intended follow-up duration of 12 months, and 11 studies had more than 24 months follow-up. Four studies ${ }^{27} 313237$ followed the postoperation patient profiles but with some reported at different time points. We included those data into the nearest stated time frame. Participants' mean age ranged from 36.1 to 48 years, and their mean BMI ranged from 41.8 to $50.1 \mathrm{~kg} / \mathrm{m}^{2}$. The missing rates varied substantially between studies during the entire follow-up, ranging from 0 to $67.0 \%$.

The proportion of patients with anaemia increased significantly by $8.4 \%$ (95\% CI $2.8 \%$ to $14.0 \%$ ) 1 year after the surgery (table 2 and figure 2, included nine studies, 3932 patients), from baseline $12.2 \%$ (95\% CI $8.8 \%$ to $15.6 \%$ ) to $20.9 \%$ (95\% CI $14.7 \%$ to $27.1 \%$ ). Prevalence of anaemia at 24 and 36 months of follow-up elevated to $25.9 \%$ (95\% CI $17.9 \%$ to $33.8 \%$ ) and $23.1 \%$ (95\% CI, $13.6 \%$ to $32.7 \%$ ), respectively. In parallel, a continuous decline in haemoglobin (11 studies, 4548 patients) and haematocrit (three studies, 620 patients) levels after surgery was also observed (see online supplementary table $1 \mathrm{~A}, \mathrm{~B}$ )

To elucidate the cause of anaemia after RYGB, iron deficiency was first investigated, with ferritin and iron levels. The proportion of study participants with low ferritin levels rose by $4.1 \%$ (95\% CI $-2.2 \%$ to $10.4 \%$ ) at 12 months after surgery, from $7.9 \%$ (95\% CI $0.95 \%$ to $14.8 \%$ ) at baseline to $13.4 \%$ (95\% CI $3.5 \%$ to $23.4 \%$ ) (figure $3 \mathrm{~A}$ and table 3 , included four studies, 685 


\begin{tabular}{|c|c|c|c|c|c|c|c|c|c|c|c|}
\hline Year & Author & Country & Study type & Procedure & $\begin{array}{l}\text { Case } \\
\text { number }\end{array}$ & $\begin{array}{l}\text { Intended } \\
\text { follow-up } \\
\text { (months) }\end{array}$ & $\begin{array}{l}\text { Mean age } \\
\text { (years) }\end{array}$ & $\begin{array}{l}\text { Male } \\
(\%)\end{array}$ & $\begin{array}{l}\text { Mean } \\
\text { BMI }\end{array}$ & $\begin{array}{l}\text { Nutrition } \\
\text { management }\end{array}$ & $\begin{array}{l}12 \text { months } \\
\text { missing rate (\%) }\end{array}$ \\
\hline 1992 & Avinoah E & Israel & Prospective & NR & 200 & 96 & 36.1 & 29.0 & NR & NR & 0 \\
\hline 1998 & Brolin RE & USA & Prospective & NR & 348 & 36 & - & - & - & Oral MVI & 12.6 \\
\hline 2006 & Madan AK & USA & Retrospective & Laparoscopy & 100 & 12 & - & - & - & NR & 64.0 \\
\hline 2009 & Toh SY & Australia & Retrospective & Laparoscopy & 121 & 12 & 48 & 36.4 & 43.1 & Oral MVI, $\mathrm{B}_{12} \#$ & 52.1 \\
\hline 2010 & Avgerinos DV & USA & Retrospective & NR & 206 & 20 & 40.8 & 19.9 & - & Oral MVI & 0 \\
\hline 2011 & von Drygalski A & USA & Prospective & Both & 1125 & 48 & 42 & 11.2 & 50.1 & Oral MVI & 67.0 \\
\hline 2011 & Cable CT & USA & Retrospective & Both & 1009 & 36 & 43.7 & 15.9 & - & Oral MVI & 28.6 \\
\hline 2012 & Blume CA & Brazil & Retrospective & NR & 170 & 36 & 39.5 & 20.0 & 48.8 & NR & 0 \\
\hline 2012 & Dallal RM & USA & Retrospective & NR & 590 & 12 & 43.2 & 17.2 & 47.7 & $\mathrm{Fe}^{\star}$ & 0 \\
\hline 2012 & Aarts EO & The Netherlands & Prospective & Laparoscopy & 377 & 12 & 43.4 & 27.1 & 46.8 & Oral MVI & 0 \\
\hline 2013 & Moizé V & Spain & Prospective & Laparoscopy & 294 & 60 & 45.2 & 23 & 47.4 & Oral MVI, $B_{12} \#$ & 0 \\
\hline 2014 & Gesquiere I & Belgium & Retrospective & Laparoscopy & 164 & 60 & 43 & 25 & 41.8 & $\mathrm{Fe}^{*}$ & 45.1 \\
\hline 2014 & Obinwanne KM & USA & Retrospective & Laparoscopy & 959 & 120 & 43.8 & 15.1 & 47.4 & Oral MVI, $\mathrm{Fe}^{*}$ & 17.9 \\
\hline 2014 & Salgado W Jr & Brazil & Retrospective & Open & 102 & 48 & 41.9 & 14.8 & 49.7 & Oral MVI & 4.9 \\
\hline 2014 & Ledoux S & France & Prospective & Laparoscopy & 144 & 36 & 42.9 & 9.7 & 48.2 & Oral MVI, $\mathrm{B}_{12}{ }^{*}$ & 0 \\
\hline
\end{tabular}

$\mathrm{Fe}^{*}$ : Therapeutic iron supplementation; $\mathrm{B}_{12}{ }^{*}$ : therapeutic intramuscular vitamin $\mathrm{B}_{12}$

$\mathrm{B}_{12} \#$ : Augmented with intramuscular vitamin $\mathrm{B}_{12}$ prophylaxis.

BMI, body mass index; NR, not reported; Oral MVI, prophylactic oral tablet with multivitamin and mineral supplement.

Table 2 Proportion of study participants with anaemia before and after RYGB surgery

\begin{tabular}{|c|c|c|c|c|c|c|c|c|c|c|c|c|c|c|c|c|}
\hline \multirow[b]{2}{*}{ Year } & \multirow[b]{2}{*}{ Author } & \multicolumn{2}{|c|}{ Before surgery } & \multicolumn{2}{|c|}{6 months } & \multicolumn{2}{|c|}{12 months } & \multicolumn{2}{|c|}{24 months } & \multicolumn{2}{|c|}{36 months } & \multirow{2}{*}{$\begin{array}{l}\Delta 6 \text { month } \\
\text { (Per cent) }\end{array}$} & \multirow{2}{*}{$\begin{array}{l}\Delta 12 \text { month } \\
\text { (Per cent) }\end{array}$} & \multirow{2}{*}{$\begin{array}{l}\Delta 24 \text { month } \\
\text { (Per cent) }\end{array}$} & \multirow{2}{*}{$\begin{array}{l}\Delta 36 \text { month } \\
\text { (Per cent) }\end{array}$} & \multirow[b]{2}{*}{ Definition of anaemia } \\
\hline & & No & Per cent & No & Per cent & No & Per cent & No & Per cent & No & Per cent & & & & & \\
\hline 2009 & Toh SY & $7 / 113$ & 6.0 & & - & $10 / 58$ & 17.0 & - & - & - & - & - & 11.0 & - & - & $\begin{array}{l}\mathrm{Hb} \text { : men }<13.0 \mathrm{~g} / \mathrm{dL} \text {; } \\
\text { women }<11.9 \mathrm{~g} / \mathrm{dL}\end{array}$ \\
\hline 2011 & von Drygalski A & $125 / 1037$ & 12.0 & $70 / 559$ & 12.5 & $43 / 371$ & 11.6 & $35 / 153$ & 22.9 & - & - & 0.5 & -0.4 & 10.9 & - & $\begin{array}{l}\mathrm{Hb}: \text { men }<13.0 \mathrm{~g} / \mathrm{dL} ; \\
\text { women }<12.0 \mathrm{~g} / \mathrm{day} \mathrm{L}\end{array}$ \\
\hline 2011 & Cable CT & $156 / 1009$ & 15.5 & - & - & $259 / 720$ & 36.0 & - & - & - & - & - & 20.5 & - & - & $\begin{array}{l}\mathrm{Hb} \text { : men }<14.0 \mathrm{~g} / \mathrm{dL} ; \\
\text { women }<12.0 \mathrm{~g} / \mathrm{dL}\end{array}$ \\
\hline 2012 & Blume CA & $11 / 170$ & 6.5 & $37 / 170$ & 21.8 & $32 / 170$ & 18.8 & $58 / 170$ & 34.1 & $57 / 170$ & 33.5 & 15.3 & 12.3 & 27.6 & 27.0 & $\begin{array}{l}\mathrm{Hb} \text { : men }<13.0 \mathrm{~g} / \text { day } \mathrm{L} ; \\
\text { women }<12.0 \mathrm{~g} / \mathrm{dL}\end{array}$ \\
\hline 2012 & Aarts EO & $27 / 377$ & 7.2 & $50 / 377$ & 13.3 & $71 / 377$ & 18.8 & - & - & - & - & 6.1 & 11.6 & - & - & $\begin{array}{l}\mathrm{Hb}: \text { men }<13.5 \mathrm{~g} / \mathrm{dL} \text {; } \\
\text { women }<11.9 \mathrm{~g} / \mathrm{dL}\end{array}$ \\
\hline 2012 & Dallal RM & $97 / 590$ & 16.4 & - & - & $162 / 590$ & 27.5 & - & - & - & - & - & 11.1 & - & - & $\begin{array}{l}\text { Hct: men }<39 \% \text {; } \\
\text { women }<35 \%\end{array}$ \\
\hline 2013 & Moizé V & $65 / 294$ & 22 & $61 / 294$ & 20.8 & $59 / 294$ & 19.9 & $46 / 259$ & 17.7 & $35 / 203$ & 17.2 & -1.2 & -2.1 & -4.3 & -4.8 & $\begin{array}{l}\mathrm{Hb}: \text { men }<13.7 \mathrm{~g} / \mathrm{dL} ; \\
\text { women }<12.2 \mathrm{~g} / \mathrm{dL}\end{array}$ \\
\hline 2014 & Salgado W Jr & $22 / 102$ & 21.6 & - & - & $25 / 97$ & 25.8 & $26 / 85$ & 30.6 & $27 / 89$ & 30.3 & - & 4.2 & 9.0 & 8.7 & $\begin{array}{l}\mathrm{Hb}: \text { men }<13 \mathrm{~g} / \mathrm{dL} \text {; } \\
\text { women }<12 \mathrm{~g} / \mathrm{dL}\end{array}$ \\
\hline 2014 & Ledoux S & $9 / 144$ & 6.0 & - & - & $17 / 144$ & 12.0 & - & - & $15 / 115$ & 13.0 & - & 6.0 & - & 7.0 & $\begin{array}{l}\mathrm{Hb} \text { : men < } 11.5 \mathrm{~g} / \mathrm{dL} ; \\
\text { women }<11.5 \mathrm{~g} / \mathrm{dL}\end{array}$ \\
\hline \multicolumn{2}{|c|}{ Summary estimate } & \multirow{2}{*}{\multicolumn{2}{|c|}{$\begin{array}{l}12.2 \\
(8.8 \text { to } 15.6)\end{array}$}} & \multirow{2}{*}{\multicolumn{2}{|c|}{$\begin{array}{l}16.6 \\
(12.1 \text { to } 21.0)\end{array}$}} & \multirow{2}{*}{\multicolumn{2}{|c|}{$\begin{array}{l}20.9 \\
\text { (14.7 to } 27.1)\end{array}$}} & \multirow{2}{*}{\multicolumn{2}{|c|}{$\begin{array}{l}25.9 \\
\text { (17.9 to } 33.8 \text { ) }\end{array}$}} & \multirow{2}{*}{\multicolumn{2}{|c|}{$\begin{array}{l}23.1 \\
\text { (13.6 to } 32.7)\end{array}$}} & 4.8 & 8.4 & 10.8 & 9.4 & \\
\hline \multicolumn{2}{|c|}{$\begin{array}{l}(95 \% \mathrm{Cl}) \\
\mathrm{I}^{2}\end{array}$} & & & & & & & & & & & $\begin{array}{l}(-1.1 \text { to } 10.8) \\
81.9\end{array}$ & $\begin{array}{l}(2.8 \text { to } 14.0) \\
88.2\end{array}$ & $\begin{array}{l}\text { (-3.3 to } 24.8) \\
91.7\end{array}$ & $\begin{array}{l}(-4.7 \text { to } 23.5) \\
91.3\end{array}$ & \\
\hline
\end{tabular}




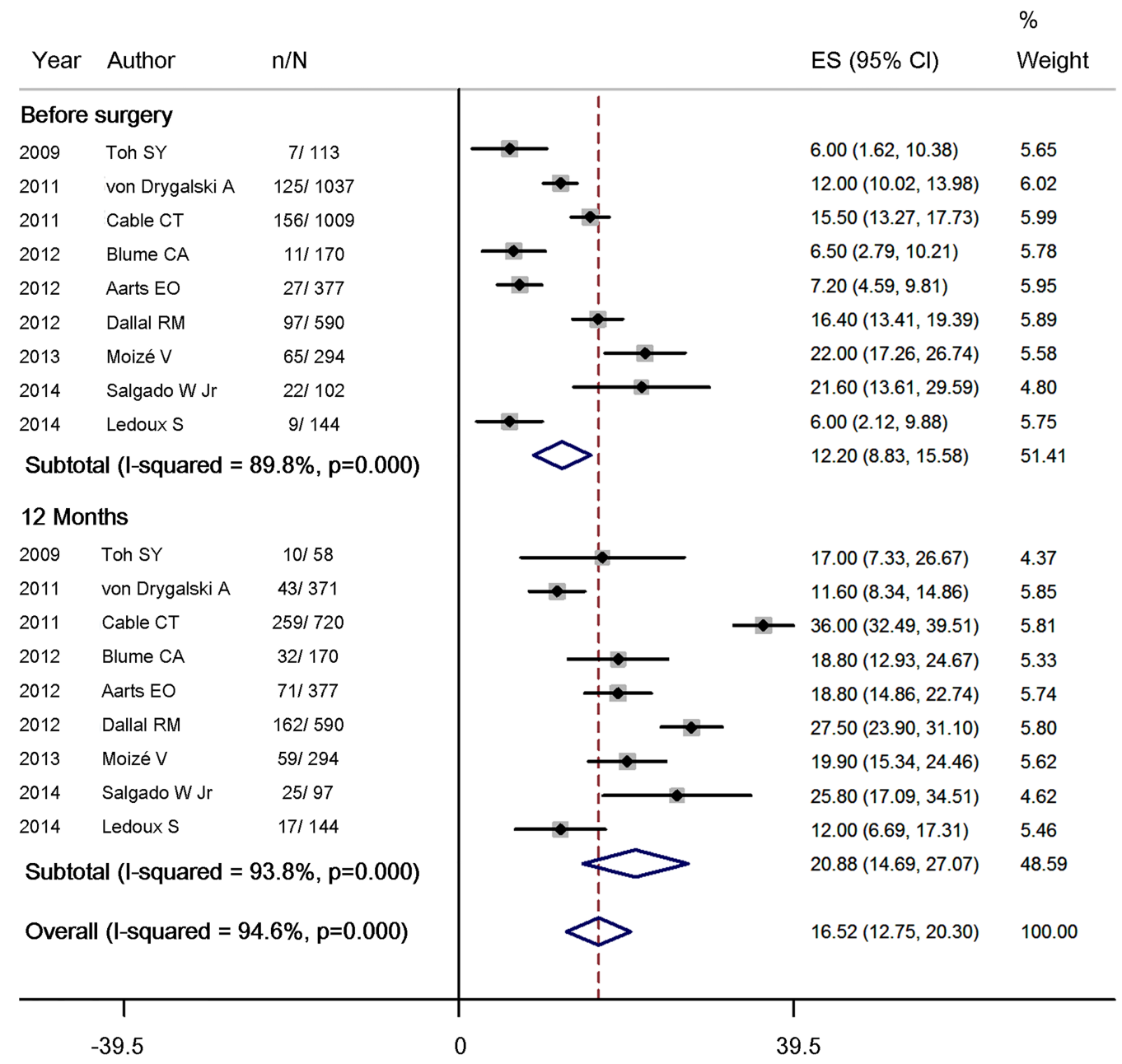

NOTE: Weights are from random effects analysis

Figure 2 Forest plot for proportion of study participants with anaemia before RYGB and 12 months after Roux-en-Y gastric bypass (RYGB).

patients). The proportion of patients with low ferritin levels rose to $23.0 \%$ (95\% CI $9.1 \%$ to $36.9 \%$ ) and $26.5 \%$ (95\% CI $20.6 \%$ to $32.4 \%)$ at 24 and 36 months, respectively. Pooled estimates for mean serum ferritin levels (see online supplementary table 2A, four studies, 1512 patients) had the baseline at $87.6 \mathrm{ng} / \mathrm{mL}$ (95\% CI 75.4 to 99.9 ), which declined to $55.5 \mathrm{ng} / \mathrm{mL}$ (95\% CI 43.0 to $68.0)$ at 24 months follow-up. In contrast, the proportion of study participants with low levels of serum iron (figure 3B and table 4, four studies, 685 patients) and mean serum iron concentration (see online supplementary table 2B, six studies, 2072 patients) did not change significantly.

The proportion of patients with low vitamin $\mathrm{B}_{12}$ levels increased $3.6 \%(95 \%$ CI $-1.2 \%$ to $8.4 \%)$ from $2.3 \%$ (95\% CI $1.1 \%$ to $3.6 \%)$ at baseline, to $6.5 \%$ (95\% CI $1.8 \%$ to $11.1 \%$ ) at 12 months of follow-up (figure $3 \mathrm{C}$ and table 5 , included five studies, 829 patients), with a consistent decrease in mean vitamin $\mathrm{B}_{12}$ levels (see online supplementary table $2 \mathrm{C}$, included seven studies, 2204 patients). A continuous increase in the proportion of patients with vitamin $\mathrm{B}_{12}$ deficiency was noted, that is,
$5.4 \%(95 \%$ CI $3.3 \%$ to $7.6 \%)$ at 24 months and $7.2 \%$ (95\% CI $4.6 \%$ to $9.7 \%$ ) at 36 months. In contrast, no obvious change was found for the proportion of patients with low folate levels (figure 3D and table 6, four studies, 535 patients), nor for mean folate levels, before and after surgery (see online supplementary table 2D, five studies, 1840 patients). Overall, the proportion of patients with anaemia and with deficiencies in iron, ferritin, folate and vitamin $B_{12}$ at different time points varied across individual studies, with $\mathrm{I}^{2}$ values raised as high as $94.8 \%$, indicating substantial heterogeneity between studies. In contrast, $\mathrm{I}^{2}$ values for ferritin, serum iron, folate and vitamin $B_{12}$ levels were almost equal to zero, which may be due to large variance for these outcomes within studies. For the proportion of patients with anaemia 12 months after surgery, publication bias was not detected by the funnel plot $(\mathrm{p}=0.754$ with Begg's test, or $\mathrm{p}=0.978$ with Egger's test) (figure 4). The number of studies for other primary outcomes was scarce ( $\leq 5$ studies), which may be statistically underpowered; these studies were thus not evaluated in this analysis. 


\section{A Ferritin}

$\%$

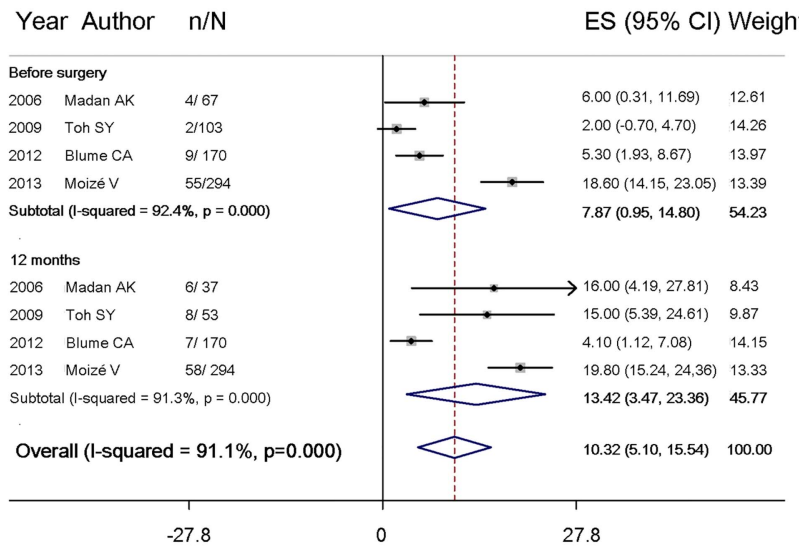

\section{Vitamin $\mathrm{B}_{12}$}

$\%$

\begin{tabular}{|c|c|c|c|c|c|}
\hline Yea & Ir Autho & $\mathrm{n} / \mathrm{N}$ & & ES (95\% Cl) & Weig \\
\hline Before & urgery & & & & \\
\hline 2006 & Madan AK & $3 / 59$ & & $\begin{array}{l}.50(-0.56,1.056) \\
1.0(-1.08,308)\end{array}$ & 6.182 \\
\hline 2009 & Toh SY & $1 / 88$ & $\div$ & & $\begin{array}{l}12.24 \\
11.52\end{array}$ \\
\hline 2012 & Blume CA & $5 / 170$ & & & \\
\hline 2013 & Moizé V & $5 / 294$ & $\rightarrow$ & $1.80(0.28,3.32)$ & 13.03 \\
\hline 2014 & Ledoux S & $7 / 144$ & & $5.00(1.44,8.56)$ & 9.77 \\
\hline Subtota & (1-squared $=$ & $\%, p=0.268)$ & $\diamond$ & $2.32(1.05,3.58)$ & 53.33 \\
\hline 12 mon & & & & & \\
\hline 2006 & Madan AK & //36 & + & $0.03(-0.52,0.57)$ & 13.93 \\
\hline 2009 & Toh SY & $6 / 56$ & & $11.00(2.81,19.19)$ & 4.22 \\
\hline 2012 & Blume CA & $6 / 170$ & $\rightarrow$ & $3.50(0.74,6.26)$ & 11.12 \\
\hline 2013 & Moizé V & $18 / 294$ & & $6.20(3.44,8.96)$ & 11.13 \\
\hline 2014 & Ledoux S & $23 / 144$ & & $\begin{array}{l}16.00(10.01,21.99) \\
\text { ) }\end{array}$ & 6.26 \\
\hline Subtota & (1-squared = & $\%, p=0.000$ ) & & $6.47(1.80,11.14)$ & 46.67 \\
\hline Over & all (I-squar & $86.9 \%, p=0.000)$ & & $4.07(2.06,6.08)$ & 100.00 \\
\hline
\end{tabular}

NOTE: Weights are from random effects analysis
B Serum Iron

$\%$

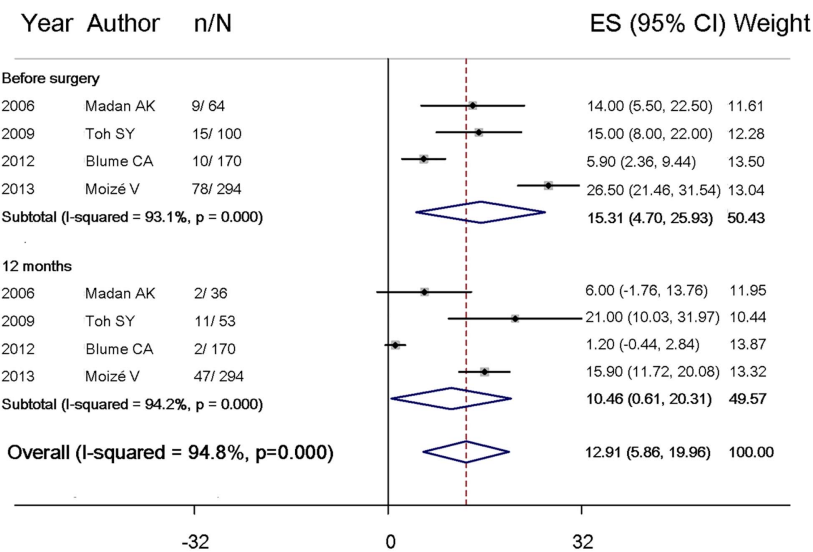

\section{Folate}

$\%$

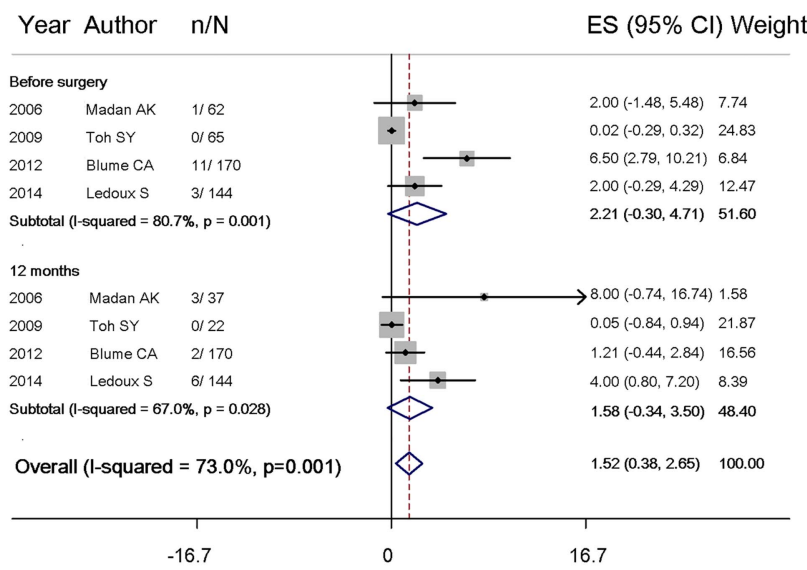

Figure 3 Forest plot for proportion of study participants with (A) ferritin (B) serum iron (C) vitamin $B_{12}$ and (D) folate deficiencies, before Roux-en-Y gastric bypass (RYGB) and 12 months after RYGB.

\section{DISCUSSION}

Several studies reported that a substantial proportion of patients already had anaemia prior to RYBG surgery, as well as having deficiencies in iron, folate and vitamin $\mathrm{B}_{12}$. This can probably be explained by the fact that obesity is a known risk factor for nutrient deficiencies despite an excessive calorie reserve. ${ }^{42-44}$ Our meta-analysis observed a nearly twofold increase in anaemia occurrence at 12 months after RYGB surgery, with an exacerbating declining of haemoglobin/haematocrit over time. However, the iron level may not decrease after RYGB and folate deficiency is correctable with oral multivitamin supplementation. It is important to intervene early on in cases of iron deficiency anaemia with ferritin profiling, and augmented supplementation is important for vitamin $B_{12}$, the deficiency of which may occur later, from 12 months postoperation on.

In our current meta-analyses, frequency of ferritin deficiency increased by twofold in patients at 6 months after surgery, and this trend became substantially aggravated at 24 and 36 months. However, no significant change in serum iron concentration after RYGB surgery was found. Since serum ferritin is a more specific and early indicator for iron deficiency anaemia, the low ferritin levels with stable serum iron concentrations suggest reduced body iron capacity. ${ }^{45}$ Although ferritin is also an acute-phase protein, and elevation of ferritin has been reported as early as the first month after surgery, ${ }^{15}$ the decreasing trend in ferritin level is observed at 6 months or longer after surgery, when no active inflammation is expected.

Reasons for iron deficiency can be attributed to the bariatric procedure of RYGB, which have dual effects on intake restriction and malabsorption. ${ }^{46}$ After RYGB surgery, patients dramatically reduce their caloric intake with concomitant changes in eating behaviour and dietary adherence. ${ }^{47}$ Postoperative changes in food preferences, including intolerance for meats and dairy products, have been reported in patients following RYGB. ${ }^{27} 48$ Hypochlorhydria, the virtual absence of hydrochloric acid secretion by the stomach, is also known to decrease the bioavailability of dietary iron. ${ }^{49}$ Furthermore, after RYGB, ingested food bypasses through most of the stomach and duodenum, as well as 
Table 3 Proportion of study participants with low ferritin level before and after RYGB surgery

\begin{tabular}{|c|c|c|c|c|c|c|c|c|c|c|c|c|c|c|c|c|}
\hline \multirow[b]{2}{*}{ Year } & \multirow[b]{2}{*}{ Author } & \multicolumn{2}{|c|}{ Before surgery } & \multicolumn{2}{|c|}{6 months } & \multicolumn{2}{|c|}{12 months } & \multicolumn{2}{|c|}{24 months } & \multicolumn{2}{|c|}{36 months } & \multirow{2}{*}{$\begin{array}{l}\Delta 6 \text { month } \\
\text { (Per cent) }\end{array}$} & \multirow{2}{*}{$\begin{array}{l}\Delta 12 \text { month } \\
\text { (Per cent) }\end{array}$} & \multirow{2}{*}{$\begin{array}{l}\Delta 24 \text { month } \\
\text { (Per cent) }\end{array}$} & \multirow{2}{*}{$\begin{array}{l}\Delta 36 \text { month } \\
\text { (Per cent) }\end{array}$} & \multirow{2}{*}{$\begin{array}{l}\text { Cut-off point in } \\
\text { ferritin level }\end{array}$} \\
\hline & & No & Per cent & No & Per cent & No & Per cent & No & Per cent & No & Per cent & & & & & \\
\hline 2006 & Madan AK & $4 / 67$ & 6.0 & $11 / 56$ & 20.0 & $6 / 37$ & 16.0 & - & - & - & - & 14.0 & 10.0 & - & - & NR \\
\hline 2009 & Toh SY & $2 / 103$ & 2.0 & - & - & $8 / 53$ & 15.0 & - & - & - & - & - & 13.0 & - & - & $<15 \mathrm{ng} / \mathrm{mL}$ \\
\hline 2012 & Blume CA & 9/170 & 5.3 & $7 / 170$ & 4.1 & $7 / 170$ & 4.1 & $27 / 170$ & 15.9 & $40 / 170$ & 23.5 & -1.2 & -1.2 & 10.6 & 18.2 & $\begin{array}{l}\text { Men <22 ng/mL; } \\
\text { Women }<10 \mathrm{ng} / \mathrm{mL}\end{array}$ \\
\hline 2013 & Moizé V & $55 / 294$ & 18.6 & $56 / 294$ & 19.0 & $58 / 294$ & 19.8 & $78 / 259$ & 30.1 & $60 / 203$ & 29.5 & 0.4 & 1.2 & 11.5 & 10.9 & $<15 \mathrm{ng} / \mathrm{mL}$ \\
\hline \multirow{3}{*}{\multicolumn{2}{|c|}{$\begin{array}{l}\text { Summary estimate } \\
(95 \% \mathrm{Cl})\end{array}$}} & \multirow{2}{*}{\multicolumn{2}{|c|}{$\begin{array}{l}7.9 \\
(0.95 \text { to } 14.8)\end{array}$}} & \multirow{2}{*}{\multicolumn{2}{|c|}{$\begin{array}{l}13.9 \\
(2.0 \text { to } 25.8)\end{array}$}} & \multirow{2}{*}{\multicolumn{2}{|c|}{$\begin{array}{l}13.4 \\
\text { (3.5 to 23.4) }\end{array}$}} & \multirow{2}{*}{\multicolumn{2}{|c|}{$\begin{array}{l}23.0 \\
(9.1 \text { to } 36.9)\end{array}$}} & \multirow{2}{*}{\multicolumn{2}{|c|}{$\begin{array}{l}26.5 \\
(20.6 \text { to } 32.4)\end{array}$}} & 2.4 & 4.1 & 11.0 & 14.7 & \\
\hline & & & & & & & & & & & & ( -4.2 to 9.0$)$ & $(-2.2$ to 10.4$)$ & (6.2 to 15.8 ) & (7.5 to 21.8 ) & \\
\hline & & \multicolumn{2}{|c|}{92.4} & \multicolumn{2}{|c|}{94.1} & \multicolumn{2}{|c|}{91.3} & \multicolumn{2}{|c|}{92.1} & \multicolumn{2}{|c|}{42.2} & 63.5 & 62.9 & 0.0 & 45.7 & \\
\hline
\end{tabular}

Table 4 Proportion of study participants with low level of serum iron before and after RYGB surgery

\begin{tabular}{|c|c|c|c|c|c|c|c|c|c|c|c|c|c|c|c|c|}
\hline \multirow[b]{2}{*}{ Year } & \multirow[b]{2}{*}{ Author } & \multicolumn{2}{|c|}{ Before surgery } & \multicolumn{2}{|c|}{6 months } & \multicolumn{2}{|c|}{12 months } & \multicolumn{2}{|c|}{24 months } & \multicolumn{2}{|c|}{36 months } & \multirow{2}{*}{$\begin{array}{l}\Delta 6 \text { month } \\
\text { (Per cent) }\end{array}$} & \multirow{2}{*}{$\begin{array}{l}\Delta 12 \text { month } \\
\text { (Per cent) }\end{array}$} & \multirow{2}{*}{$\begin{array}{l}\Delta 24 \text { month } \\
\text { (Per cent) }\end{array}$} & \multirow{2}{*}{$\begin{array}{l}\Delta 36 \text { month } \\
\text { (Per cent) }\end{array}$} & \multirow{2}{*}{$\begin{array}{l}\text { Cut-off point in } \\
\text { serum iron leve }\end{array}$} \\
\hline & & No. & Per cent & No & Per cent & No & Per cent & No & Per cent & No & Per cent & & & & & \\
\hline 2006 & Madan AK & 9/64 & 14.0 & $6 / 56$ & 10.0 & $2 / 36$ & 6.0 & - & - & - & - & -4.0 & -8.0 & - & - & NR \\
\hline 2009 & Toh SY & $15 / 100$ & 15.0 & - & - & $11 / 53$ & 21.0 & - & - & - & - & - & 6.0 & - & - & $<50 \mu \mathrm{g} / \mathrm{dL}$ \\
\hline 2012 & Blume CA & $10 / 170$ & 5.9 & $10 / 170$ & 5.9 & $2 / 170$ & 1.2 & $15 / 170$ & 8.8 & $14 / 170$ & 8.2 & 0 & -4.7 & 2.9 & 2.3 & $\begin{array}{l}\text { Men }<49 \mu \mathrm{g} / \mathrm{dL} \\
\text { women }<37 \mu \mathrm{g} / \mathrm{dL}\end{array}$ \\
\hline 2013 & Moizé V & $78 / 294$ & 26.5 & $54 / 294$ & 18.2 & $47 / 294$ & 15.9 & $27 / 259$ & 10.6 & $25 / 203$ & 12.5 & -8.3 & -10.6 & -15.9 & -14.0 & $<50 \mu \mathrm{g} / \mathrm{dL}$ \\
\hline \multirow{2}{*}{\multicolumn{2}{|c|}{$\begin{array}{l}\text { Summary estimate } \\
(95 \% \mathrm{Cl})\end{array}$}} & \multirow{2}{*}{\multicolumn{2}{|c|}{$\begin{array}{l}15.3 \\
(4.7 \text { to } 25.9)\end{array}$}} & \multirow{2}{*}{\multicolumn{2}{|c|}{$\begin{array}{l}11.4 \\
(2.8 \text { to } 20.0)\end{array}$}} & \multirow{2}{*}{\multicolumn{2}{|c|}{$\begin{array}{l}10.5 \\
(0.6 \text { to } 20.3)\end{array}$}} & \multirow{2}{*}{\multicolumn{2}{|c|}{$\begin{array}{l}9.8 \\
(7.0 \text { to } 12.6)\end{array}$}} & \multirow{2}{*}{\multicolumn{2}{|c|}{$\begin{array}{l}10.2 \\
(6.0 \text { to } 14.4)\end{array}$}} & -3.7 & -5.6 & -6.4 & -5.7 & \\
\hline & & & & & & & & & & & & (-9.4 to 2.0 ) & $(-10.8$ to -0.4$)$ & (-24.9 to 12.0$)$ & (-21.7 to 10.3$)$ & \\
\hline \multicolumn{2}{|c|}{$1^{2}$} & \multicolumn{2}{|c|}{93.1} & \multicolumn{2}{|c|}{89.0} & \multicolumn{2}{|c|}{94.2} & \multicolumn{2}{|c|}{0.0} & \multicolumn{2}{|c|}{46.9} & 47.5 & 47.0 & 94.8 & 92.6 & \\
\hline
\end{tabular}

NR, not reported; RYGB, Roux-en-Y gastric bypass.

Table 5 Proportion of study participants with vitamin $B_{12}$ deficiency before and after RYGB surgery

\begin{tabular}{|c|c|c|c|c|c|c|c|c|c|c|c|c|c|c|c|c|}
\hline \multirow[b]{2}{*}{ Year } & \multirow[b]{2}{*}{ Author } & \multicolumn{2}{|c|}{ Before surgery } & \multicolumn{2}{|c|}{6 months } & \multicolumn{2}{|c|}{12 months } & \multicolumn{2}{|c|}{24 months } & \multicolumn{2}{|c|}{36 months } & \multirow{2}{*}{$\begin{array}{l}\Delta 6 \text { month } \\
\text { (Per cent) }\end{array}$} & \multirow{2}{*}{$\begin{array}{l}\Delta 12 \text { month } \\
\text { (Per cent) }\end{array}$} & \multirow{2}{*}{$\begin{array}{l}\Delta 24 \text { month } \\
\text { (Per cent ) }\end{array}$} & \multirow{2}{*}{$\begin{array}{l}\Delta 36 \text { month } \\
\text { (Per cent ) }\end{array}$} & \multirow{2}{*}{$\begin{array}{l}\text { Definition of } \\
\text { deficiency }\end{array}$} \\
\hline & & No & Per cent & No & Per cent & No & Per cent & No & Per cent & No & Per cent & & & & & \\
\hline 2006 & Madan AK & $3 / 59$ & 5.0 & $0 / 56$ & $0^{*}$ & $0 / 36$ & $0^{*}$ & - & - & - & - & -5.0 & -5.0 & - & - & NR \\
\hline 2009 & Toh SY & $1 / 88$ & 1.0 & - & - & $6 / 56$ & 11.0 & - & - & - & - & - & 10.0 & - & - & $<196 \mathrm{pg} / \mathrm{mL}$ \\
\hline 2012 & Blume CA & $5 / 170$ & 2.9 & $6 / 170$ & 3.5 & $6 / 170$ & 3.5 & 9/170 & 5.3 & $12 / 170$ & 7.1 & 0.6 & 0.6 & 2.4 & 4.2 & $<211 \mathrm{pg} / \mathrm{mL}$ \\
\hline 2013 & Moizé V & $5 / 294$ & 1.8 & 9/294 & 2.9 & $18 / 294$ & 6.2 & $14 / 259$ & 5.5 & $12 / 203$ & 5.8 & 1.1 & 4.4 & 3.7 & 4.0 & $<250 \mathrm{pg} / \mathrm{mL}$ \\
\hline 2014 & Ledoux S & $7 / 144$ & 5.0 & - & - & $23 / 144$ & 16.0 & - & - & $13 / 115$ & 11.0 & - & 11.0 & - & 6.0 & $<190 \mathrm{pg} / \mathrm{mL}$ \\
\hline \multicolumn{2}{|c|}{$\begin{array}{l}\text { Summary estimate } \\
(95 \% \mathrm{Cl})\end{array}$} & \multicolumn{2}{|c|}{$\begin{array}{l}2.3 \\
(1.1 \text { to } 3.6)\end{array}$} & \multicolumn{2}{|c|}{$\begin{array}{l}1.9 \\
(0 \text { to } 4.4) \dagger\end{array}$} & \multicolumn{2}{|c|}{$\begin{array}{l}6.5 \\
(1.8 \text { to } 11.1)\end{array}$} & \multicolumn{2}{|c|}{$\begin{array}{l}5.4 \\
(3.3 \text { to } 7.6)\end{array}$} & \multicolumn{2}{|c|}{$\begin{array}{l}7.2 \\
(4.6 \text { to } 9.7)\end{array}$} & \multicolumn{2}{|c|}{$\begin{array}{l}-0.3 \\
(-3.3 \text { to } 2.7)\end{array}$} & $\begin{array}{l}3.6 \\
(-1.2 \text { to } 8.4) \\
77.7\end{array}$ & $\begin{array}{l}3.2 \\
(0.7 \text { to } 5.8) \\
0.0\end{array}$ & $\begin{array}{l}4.4 \\
(1.8 \text { to } 7.0) \\
0.0\end{array}$ \\
\hline
\end{tabular}

${ }^{*}$ Data were included in the analysis with a continuity correction factor of 0.01

†The lower limits of $95 \% \mathrm{Cl}$ were truncated as zero due to negative values.

NR, not reported; RYGB, Roux-en-Y gastric bypass. 

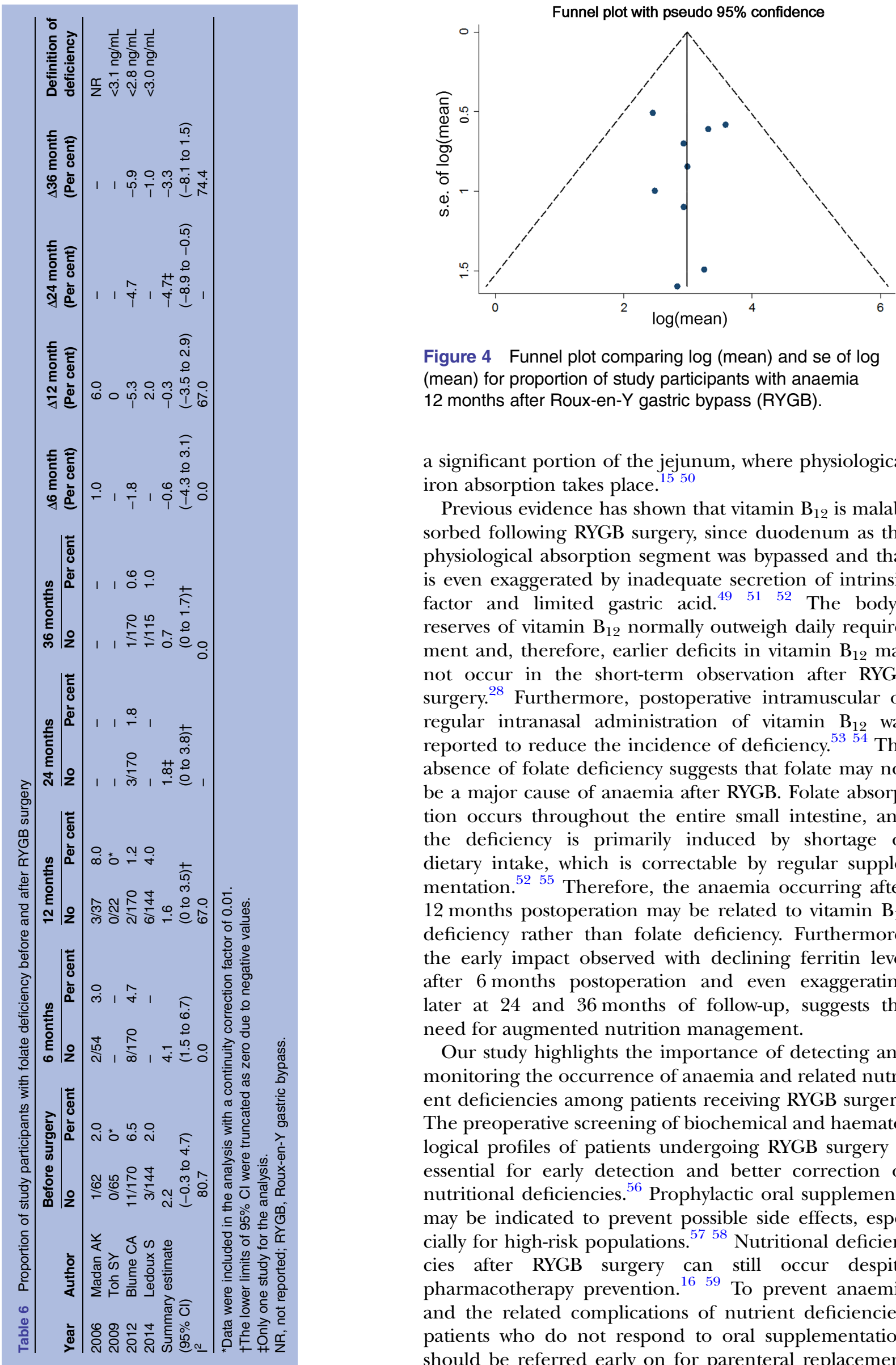

Figure 4 Funnel plot comparing log (mean) and se of log (mean) for proportion of study participants with anaemia 12 months after Roux-en-Y gastric bypass (RYGB).

a significant portion of the jejunum, where physiological iron absorption takes place. ${ }^{15} 50$

Previous evidence has shown that vitamin $\mathrm{B}_{12}$ is malabsorbed following RYGB surgery, since duodenum as the physiological absorption segment was bypassed and that is even exaggerated by inadequate secretion of intrinsic factor and limited gastric acid. ${ }^{49} 51 \quad 52$ The body's reserves of vitamin $B_{12}$ normally outweigh daily requirement and, therefore, earlier deficits in vitamin $B_{12}$ may not occur in the short-term observation after RYGB surgery. ${ }^{28}$ Furthermore, postoperative intramuscular or regular intranasal administration of vitamin $B_{12}$ was reported to reduce the incidence of deficiency. ${ }^{53} 54$ The absence of folate deficiency suggests that folate may not be a major cause of anaemia after RYGB. Folate absorption occurs throughout the entire small intestine, and the deficiency is primarily induced by shortage of dietary intake, which is correctable by regular supplementation. ${ }^{52} 55$ Therefore, the anaemia occurring after 12 months postoperation may be related to vitamin $B_{12}$ deficiency rather than folate deficiency. Furthermore, the early impact observed with declining ferritin level after 6 months postoperation and even exaggerating later at 24 and 36 months of follow-up, suggests the need for augmented nutrition management.

Our study highlights the importance of detecting and monitoring the occurrence of anaemia and related nutrient deficiencies among patients receiving RYGB surgery. The preoperative screening of biochemical and haematological profiles of patients undergoing RYGB surgery is essential for early detection and better correction of nutritional deficiencies. ${ }^{56}$ Prophylactic oral supplements may be indicated to prevent possible side effects, especially for high-risk populations. ${ }^{57}$ Nutritional deficiencies after RYGB surgery can still occur despite pharmacotherapy prevention. ${ }^{16} 59$ To prevent anaemia and the related complications of nutrient deficiencies, patients who do not respond to oral supplementation should be referred early on for parenteral replacement 
therapy. ${ }^{38}$ Mean corpuscular volume, and reticulocyte and a simple blood smear, may be useful in differential diagnosis of anaemia, and bone marrow biopsy is warranted to investigate the aetiology of persistent anaemia associated with cytopaenia. ${ }^{22}{ }^{60}$ Long-term consistent monitoring of nutritional complications secondary to RYGB surgery is recommended. ${ }^{23} 58$

This is a systematic review of studies published in English (ie, single language) to look at the effect of RYGB surgery on anaemia. The study benefits from the inclusion of a large number of patients, and the results showed there is not much uncertainty about the effect of the surgery on anaemia. However, there is no formal quality assessment, and the publication bias was only evaluated for the proportion of study participants with anaemia 12 months after RYGB. Most of the included studies were retrospective in nature and some of them had high loss-to-follow-up rates or incomplete ascertainment of nutrient deficiencies among participants. Second, there were substantial differences in preoperative prophylactic and postoperative nutritional management across studies, and the heterogeneity of RYGB procedures carried out by laparotomy or laparoscopy with differences in the length of the Roux-en-Y loop. Third, monitoring of patients and the quality in reporting outcomes of interest varied across studies. We did not define specific cut-off values for our outcomes of interest because different criteria were used in different studies. Although such discrepancies reflect clinical practice, this may increase variation of summary estimates. However, we got consistent findings either based on dichotomous outcomes or continuous variables (such as \% of study participants with anaemia and $\mathrm{Hb} / \mathrm{Hct}$ levels), which supported the robust estimate in our research. Fourth, other factors related to anaemia, such as vitamins $\mathrm{B}_{1}, \mathrm{~A}, \mathrm{D}, \mathrm{E}$ and $\mathrm{K}$, and trace elements of copper and zinc, ${ }^{61-63}$ as well as changes in inflammation levels, ${ }^{60}{ }^{64-66}$ were not assessed due to lack of data in the included studies. Fifth, this study was susceptible to inherent drawbacks that reflect the challenges of using tertiary data for meta-analysis. For example, although we observed profound changes in the proportion of patients with anaemia, ferritin and vitamin $\mathrm{B}_{12}$ deficiencies after surgery, the missing rate for each outcome at 12, 24 and 36 months obviously varied. This may result in different studies being enrolled at individual time points, increase the complexity of evaluating changes in outcome measurements over time, and induce substantial heterogeneities in summary estimates. Also, as mentioned above, none of the included studies provided the variance for changes of each outcome from baseline. This precluded the accurate calculation of covariance of outcomes between different time points and led to overestimation of the variability for changes of anaemia status and related nutrient deficiencies from baseline. Finally, limited data from published reports may still be insufficient to precisely estimate the cumulative incidence of anaemia and related nutrient deficiencies, due to limited sample size and follow-up time. As more data become available through well designed and properly conducted large observational studies with long duration, more hypotheses about haematological and nutritional complications following RYGB surgery can be explored in the future.

\section{CONCLUSIONS}

Morbid obesity, although modifiable under bariatric surgery, is a life-long disease. Even with supplementation, anaemia and deficiencies of iron and vitamin $\mathrm{B}_{12}$ may develop as early as 6 months postoperatively and exacerbate over time. Anaemia may occur with declining body reserve of vitamin $\mathrm{B}_{12}$ and deteriorating iron metabolism, which can be identified early by ferritin levels, but can also be masked by those of serum iron and declining body reserve of vitamin $\mathrm{B}_{12}$. The possible risk of haematological and nutritional sequelae should be taken into consideration in clinical practice. Regular surveillance of all RYGB candidate and persistent follow-up with comprehensive nutrient profiles is strongly recommended to improve the overall health status of individuals with morbid obesity.

\section{Author affiliations}

${ }^{1}$ Department of Medicine, College of Medicine, National Taiwan University, Taipei, Taiwan

${ }^{2}$ Department of Internal Medicine, National Taiwan University Hospital, Taipei, Taiwan

${ }^{3}$ Institute of Preventive Medicine, College of Public Health, National Taiwan University, Taipei, Taiwan

${ }^{4}$ Division of Pharmacoepidemiology and Pharmacoeconomics, Department of Medicine, Brigham and Women's Hospital, Boston, Massachusetts, USA ${ }^{5}$ Graduate Institute of Medical Genomics and Proteomics, National Taiwan University, Taipei, Taiwan

Contributors T-CW, C-HC and L-MC conceived the review and developed the review protocol. T-CW, C-HC and $\mathrm{Y}-\mathrm{HD}$ were involved in data extraction. $\mathrm{Y}-\mathrm{HD}$ and $\mathrm{C}-\mathrm{HC}$ analysed the data. T-CW, C-HC, Y-HD, Y-CC and L-MC, reviewed and contributed to the manuscript drafted by T-CW and C-HC. All the authors critically revised the manuscript and agreed on the final version. L-MC is guarantor.

Funding This research received no specific grant from any funding agency in the public, commercial or not-for-profit sectors.

Competing interests None declared.

Provenance and peer review Not commissioned; externally peer reviewed.

Data sharing statement Extra data can be accessed via the Dryad data repository at http://datadryad.org/ with the doi:10.5061/dryad.6v5tn.

Open Access This is an Open Access article distributed in accordance with the Creative Commons Attribution Non Commercial (CC BY-NC 4.0) license, which permits others to distribute, remix, adapt, build upon this work noncommercially, and license their derivative works on different terms, provided the original work is properly cited and the use is non-commercial. See: http:// creativecommons.org/licenses/by-nc/4.0/

\section{REFERENCES}

1. Wang YC, McPherson K, Marsh T, et al. Health and economic burden of the projected obesity trends in the USA and the UK. Lancet 2011;378:815-25.

2. Flegal KM, Carroll MD, Kit BK, et al. Prevalence of obesity and trends in the distribution of body mass index among US adults, 1999-2010. JAMA 2012;307:491-7. 
3. Swinburn BA, Sacks G, Hall KD, et al. The global obesity pandemic: shaped by global drivers and local environments. Lancet 2011;378:804-14.

4. Svetkey LP, Stevens VJ, Brantley PJ, et al. Comparison of strategies for sustaining weight loss: the weight loss maintenance randomized controlled trial. JAMA 2008;299:1139-48.

5. Clifton PM. Dietary treatment for obesity. Nat Clin Pract Gastroenterol Hepatol 2008;5:672-81.

6. Ikramuddin S, Korner J, Lee WJ, et al. Roux-en- $Y$ gastric bypass vs intensive medical management for the control of type 2 diabetes, hypertension, and hyperlipidemia: the Diabetes Surgery Study randomized clinical trial. JAMA 2013;309:2240-9.

7. Sjostrom L, Lindroos AK, Peltonen M, et al. Lifestyle, diabetes, and cardiovascular risk factors $10 y e a r s$ after bariatric surgery. $N$ Engl $J$ Med 2004;351:2683-93.

8. Shinogle JA, Owings MF, Kozak LJ. Gastric bypass as treatment for obesity: trends, characteristics, and complications. Obes Res 2005;13:2202-9.

9. Adams TD, Gress RE, Smith SC, et al. Long-term mortality after gastric bypass surgery. N Engl J Med 2007;357:753-61.

10. Maggard MA, Shugarman LR, Suttorp M, et al. Meta-analysis: surgical treatment of obesity. Ann Intern Med 2005;142:547-59.

11. Chang SH, Stoll CR, Song J, et al. The effectiveness and risks of bariatric surgery: an updated systematic review and meta-analysis, 2003-2012. JAMA Surg 2014;149:275-87.

12. Santry HP, Gillen DL, Lauderdale DS. Trends in bariatric surgical procedures. JAMA 2005;294:1909-17.

13. Buchwald H, Oien DM. Metabolic/bariatric surgery worldwide 2011. Obes Surg 2013;23:427-36.

14. Bal B, Koch TR, Finelli FC, et al. Managing medical and surgical disorders after divided Roux-en-Y gastric bypass surgery. Nat Rev Gastroenterol Hepatol 2010;7:320-34.

15. von Drygalski A, Andris DA. Anemia after bariatric surgery: more than just iron deficiency. Nutr Clin Pract 2009:24:217-26.

16. Gasteyger C, Suter M, Gaillard RC, et al. Nutritional deficiencies after Roux-en-Y gastric bypass for morbid obesity often cannot be prevented by standard multivitamin supplementation. Am J Clin Nutr 2008;87:1128-33

17. Malinowski SS. Nutritional and metabolic complications of bariatric surgery. Am J Med Sci 2006;331:219-25.

18. Marinella MA. Anemia following Roux-en-Y surgery for morbid obesity: a review. South Med J 2008;101:1024-31.

19. Liberati A, Altman DG, Tetzlaff J, et al. The PRISMA statement for reporting systematic reviews and meta-analyses of studies that evaluate healthcare interventions: explanation and elaboration. $B M J$ 2009;339:b2700.

20. Stroup DF, Berlin JA, Morton SC, et al. Meta-analysis of observational studies in epidemiology: a proposal for reporting. Meta-analysis Of Observational Studies in Epidemiology (MOOSE) group. JAMA 2000;283:2008-12.

21. Love AL, Billett $\mathrm{HH}$. Obesity, bariatric surgery, and iron deficiency: true, true, true and related. Am J Hematol 2008;83:403-9.

22. Poitou Bernert $\mathrm{C}$, Ciangura $\mathrm{C}$, Coupaye $\mathrm{M}$, et al. Nutritiona deficiency after gastric bypass: diagnosis, prevention and treatment. Diabetes Metab 2007;33:13-24

23. Ziegler O, Sirveaux MA, Brunaud L, et al. Medical follow up after bariatric surgery: nutritional and drug issues. General recommendations for the prevention and treatment of nutritional deficiencies. Diabetes Metab 2009;35(6 Pt 2):544-57.

24. Salameh BS, Khoukaz MT, Bell RL. Metabolic and nutritional changes after bariatric surgery. Expert Rev Gastroenterol Hepatol 2010;4:217-23.

25. Fujioka K. Follow-up of nutritional and metabolic problems after bariatric surgery. Diabetes care 2005;28:481-4

26. Jauregui-Lobera I. Iron deficiency and bariatric surgery. Nutrients 2013:5:1595-608.

27. Avinoah E, Ovnat A, Charuzi I. Nutritional status seven years after Roux-en-Y gastric bypass surgery. Surgery 1992;111:137-42.

28. Brolin RE, Gorman JH, Gorman RC, et al. Are vitamin B12 and folate deficiency clinically important after roux-en-Y gastric bypass? $J$ Gastrointest Surg 1998;2:436-42.

29. Madan AK, Orth WS, Tichansky DS, et al. Vitamin and trace minera levels after laparoscopic gastric bypass. Obes Surg 2006;16:603-6.

30. Toh SY, Zarshenas N, Jorgensen J. Prevalence of nutrient deficiencies in bariatric patients. Nutrition 2009;25:1150-6.

31. Avgerinos DV, Llaguna $\mathrm{OH}$, Seigerman $\mathrm{M}$, et al. Incidence and risk factors for the development of anemia following gastric bypass surgery. World J Gastroenterol 2010;16:1867-70.

32. von Drygalski A, Andris DA, Nuttleman PR, et al. Anemia after bariatric surgery cannot be explained by iron deficiency alone: results of a large cohort study. Surg Obes Relat Dis 2011;7:151-6.
33. Cable CT, Colbert CY, Showalter T, et al. Prevalence of anemia after Roux-en-Y gastric bypass surgery: what is the right number? Surg Obes Relat Dis 2011;7:134-9.

34. Blume CA, Boni CC, Casagrande DS, et al. Nutritional profile of patients before and after Roux-en-Y gastric bypass: 3-year follow-up. Obes Surg 2012;22:1676-85.

35. Dallal RM, Leighton J, Trang A. Analysis of leukopenia and anemia after gastric bypass surgery. Surg Obes Relat Dis 2012;8:164-8.

36. Aarts EO, van Wageningen B, Janssen IM, et al. Prevalence of anemia and related deficiencies in the first year following laparoscopic gastric bypass for morbid obesity. $J$ Obes 2012;2012:193705.

37. Moize $\mathrm{V}$, Andreu A, Flores L, et al. Long-term dietary intake and nutritional deficiencies following sleeve gastrectomy or Roux-En-Y gastric bypass in a Mediterranean population. J Acad Nutr Diet 2013;113:400-10

38. Gesquiere I, Lannoo M, Augustijns $\mathrm{P}$, et al. Iron deficiency after Roux-en-Y gastric bypass: insufficient iron absorption from oral iron supplements. Obes Surg 2014;24:56-61.

39. Obinwanne KM, Fredrickson KA, Mathiason MA, et al. Incidence, treatment, and outcomes of iron deficiency after laparoscopic Roux-en-Y gastric bypass: a 10-year analysis. J Am Coll Surg 2014:218:246-52

40. Salgado W Jr, Modotti C, Nonino CB, et al. Anemia and iron deficiency before and after bariatric surgery. Surg Obes Relat Dis 2014:10:49-54.

41. Ledoux S, Calabrese D, Bogard C, et al. Long-term evolution of nutritional deficiencies after gastric bypass: an assessment according to compliance to medical care. Ann Surg 2014;259:1104-10.

42. Flancbaum L, Belsley S, Drake V, et al. Preoperative nutritional status of patients undergoing Roux-en-Y gastric bypass for morbid obesity. J Gastrointest Surg 2006;10:1033-7.

43. Moize V, Deulofeu R, Torres F, et al. Nutritional intake and prevalence of nutritional deficiencies prior to surgery in a Spanish morbidly obese population. Obes Surg 2011;21:1382-8.

44. Aasheim ET, Hofso D, Hjelmesaeth $\mathrm{J}$, et al. Vitamin status in morbidly obese patients: a cross-sectional study. Am J Clin Nutr 2008;87:362-9.

45. Goddard AF, James MW, McIntyre AS, et al. Guidelines for the management of iron deficiency anaemia. GUT 2011;60:1309-16.

46. Blackburn GL, Hutter MM, Harvey AM, et al. Expert panel on weight loss surgery: executive report update. Obesity (Silver Spring) 2009:17:842-62.

47. Sarwer DB, Dilks RJ, West-Smith L. Dietary intake and eating behavior after bariatric surgery: threats to weight loss maintenance and strategies for success. Surg Obes Relat Dis 2011;7:644-51.

48. Balsiger BM, Kennedy FP, Abu-Lebdeh HS, et al. Prospective evaluation of Roux-en- $Y$ gastric bypass as primary operation for medically complicated obesity. Mayo Clin Proc 2000;75:673-80.

49. Smith CD, Herkes SB, Behrns KE, et al. Gastric acid secretion and vitamin B12 absorption after vertical Roux-en-Y gastric bypass for morbid obesity. Ann Surg 1993;218:91-6.

50. Cummings DE, Overduin J, Foster-Schubert KE. Gastric bypass for obesity: mechanisms of weight loss and diabetes resolution. J Clin Endocrinol Metab 2004;89:2608-15.

51. Behrns KE, Smith CD, Sarr MG. Prospective evaluation of gastric acid secretion and cobalamin absorption following gastric bypass for clinically severe obesity. Dig Dis Sci 1994;39:315-20.

52. Majumder S, Soriano J, Louie Cruz A, et al. Vitamin B12 deficiency in patients undergoing bariatric surgery: preventive strategies and key recommendations. Surg Obes Relat Dis 2013;9:1013-19.

53. Clements RH, Katasani VG, Palepu R, et al. Incidence of vitamin deficiency after laparoscopic Roux-en- $Y$ gastric bypass in a university hospital setting. Am Surg 2006;72:1196-202; discussion 203-4.

54. Mechanick JI, Youdim A, Jones DB, et al. Clinical practice guidelines for the perioperative nutritional, metabolic, and nonsurgical support of the bariatric surgery patient-2013 update: cosponsored by American Association of Clinical Endocrinologists, The Obesity Society, and American Society for Metabolic \& Bariatric Surgery. Obesity (Silver Spring, Md) 2013;21(Suppl 1):S1-27.

55. Ernst B, Thurnheer M, Schmid SM, et al. Evidence for the necessity to systematically assess micronutrient status prior to bariatric surgery. Obes Surg 2009;19:66-73.

56. Malone M. Recommended nutritional supplements for bariatric surgery patients. Ann Pharmacother 2008;42:1851-8.

57. Fried M, Yumuk V, Oppert JM, et al. Interdisciplinary European guidelines on metabolic and bariatric surgery. Obesity surgery 2014;24:42-55. 
58. Levinson R, Silverman JB, Catella JG, et al. Pharmacotherapy prevention and management of nutritional deficiencies post Roux-en-Y gastric bypass. Obes Surg 2013;23: 992-1000.

59. Chen M, Krishnamurthy A, Mohamed AR, et al. Hematological disorders following gastric bypass surgery: emerging concepts of the interplay between nutritional deficiency and inflammation. Biomed Res Int 2013;2013:205467.

60. Green R. Anemias beyond B12 and iron deficiency: the buzz about other B's, elementary, and nonelementary problems. Hematology Am Soc Hematol Educ Program 2012;2012:492-8.

61. Gletsu-Miller N, Broderius M, Frediani JK, et al. Incidence and prevalence of copper deficiency following roux-en-y gastric bypass surgery. Int J Obes (Lond) 2012;36:328-35.
62. Gletsu-Miller N, Wright BN. Mineral malnutrition following bariatric surgery. Adv Nutr 2013;4:506-17.

63. Morinigo R, Casamitjana R, Delgado S, et al. Insulin resistance, inflammation, and the metabolic syndrome following Roux-en-Y gastric bypass surgery in severely obese subjects. Diabetes care 2007;30:1906-8.

64. Cintra W, Modolin M, Faintuch J, et al. C-reactive protein decrease after postbariatric abdominoplasty. Inflammation 2012;35:316-20.

65. Rojas P, Carrasco F, Codoceo J, et al. Trace element status and inflammation parameters after 6months of Roux-en-Y gastric bypass. Obes Surg 2011;21:561-8.

66. Joao Cabrera E, Valezi AC, Delfino VD, et al. Reduction in plasma levels of inflammatory and oxidative stress indicators after Roux-en-Y gastric bypass. Obes Surg 2010;20:42-9. 